\title{
Desafíos y Oportunidades para la Diversidad del Audiovisual en Internet ${ }^{1}$
}

Trinidad García Leiva ${ }^{2}$

\section{Resumen}

A las puertas del primer cuarto del siglo XXI ya nadie cuestiona que la cadena de valor de la industria audiovisual se ha transformando. La era digital exhibe a la vez posibilidades de enriquecimiento cultural pero también despliega nuevos desafíos. Después de ofrecer un retrato general de las industrias audiovisuales en la era digital, en términos de agentes y lógicas en tensión, y partiendo del caso español, se presenta un análisis de las ventajas y desventajas que existen para la diversidad de la producción, distribución y consumo de audiovisual en Internet. Se parte de la idea de que la existencia de un audiovisual en línea diverso no está asegurada, ya que la fórmula que ha permitido que algunos agentes se convirtieran en exitosos se basa en modelos cerrados de comercialización de contenidos que añaden también restricciones a la reproducción y circulación por parte de y entre los usuarios. El objetivo último del presente texto es presentar una relación de elementos que dificultan el fortalecimiento de la diversidad de la industria audiovisual en el entorno digital. Las barreras a superar se clasifican como tecnológicas, económicas, sociales, legales y políticas.

Palabras clave: Comunicación audiovisual. Diversidad cultural. Políticas públicas. UNESCO.

\section{Introducción}

A las puertas del primer cuarto del siglo XXI ya nadie cuestiona que la cadena de valor de la industria audiovisual se ha transformando a partir de la aparición de nuevas lógicas y la consecuente redefinición de los agentes que las hacen posibles.

I Este artículo se enmarca en el proyecto "Diversidad de la industria audiovisual en la era digital" (CSO20 I452354-R) financiado por el Programa Estatal de I+D+i Orientada a los Retos de la Sociedad del Ministerio de Economía y Competitividad de España (ver: www.diversidadaudiovisual.org).

2 Profesora en la Universidad Carlos III de Madrid, donde forma parte del grupo de investigación TECMERIN. E-mail:mtgleiva@hum.uc3m.es. 
En este contexto, en el caso concreto de la influencia que ejerce Internet, los discursos utópicos suelen oponerse a los pesimistas, de forma tal que por un lado las comunidades de usuarios, libres e interconectadas, contribuyen a la realización de una "aldea global" horizontal y democrática, mientras que por otro lado esas mismas comunidades son objeto de una homogeneización y mercantilización creciente que conduce a un McWorld (Pager y Candeub, 2012). La realidad es por supuesto más compleja y las evidencias para apoyar una u otra tesis no son concluyentes.

La era digital presenta a la vez posibilidades de enriquecimiento cultural pero también despliega nuevos desafíos (Bustamante, 2016). En palabras de Freedman (2012), es posible identificar (y hay que matizar o desmontar) los discursos que proclaman que Internet facilita una economía basada en nichos y no en mercados masivos, en la flexibilidad y no en la estandarización, en la abundancia y no en la escasez, y en las start-ups y no en el tipo de corporaciones industriales que dominaron el siglo XX.

Este trabajo persigue indagar sobre cuáles son tales oportunidades y desafíos para el caso concreto del audiovisual. Se parte de la idea de que la existencia de un audiovisual en línea diverso no está asegurada, ya que la fórmula que ha permitido que algunos agentes se convirtieran en exitosos se basa en modelos cerrados de comercialización trasnacional de contenidos que añaden también restricciones a la reproducción y circulación por parte de y entre los usuarios.

Después de clarificar los conceptos centrales sobre los que se basa este artículo, se ofrece un retrato general de la industria audiovisual en Internet, en clave española y en términos de agentes y lógicas en tensión, para presentar algunas notas sobre las ventajas y desventajas que existen para la diversidad de la producción, distribución y consumo de audiovisual en línea. El objetivo es conocer cuáles son las barreras a superar.

\section{Audiovisual digital y diversidad: un debate y una definición necesarias}

Durante los últimos lustros se han producido reflexiones y debates que ponen sobre la mesa la necesidad de preservar y fomentar la diversidad de los bienes y servicios culturales, especialmente en el entorno digital. Los Estados, 
de hecho, han visto reafirmada su capacidad para formular políticas públicas en esta dirección bajo el amparo de la Declaración Universal sobre Diversidad Cultural y la Convención sobre la protección y promoción de la diversidad de las expresiones culturales, aprobadas por la Organización de las Naciones Unidas para la Educación, la Ciencia y la Cultura (UNESCO), respectivamente, en 2001 y 2005.

En el caso de este último tratado, la diversidad de y en las industrias culturales - donde el sector audiovisual tiene un papel preponderante - es el foco principal. Sin embargo la definición y aplicación de la noción de diversidad distan mucho de ser unívocas. Como analizamos en un trabajo reciente (Albornoz y García Leiva, 2017), no es simple responder qué es la diversidad y por qué esta es deseable, ya que su omnipresencia abarca ámbitos muy distintos y alimenta discursos que muchas veces apuntan en direcciones opuestas (De Beukelaer, Pyykkönen y Singh, 2015).

Si bien nadie discutiría que la diversidad de las expresiones culturales es algo que debe ser celebrado y protegido, existe debate acerca de cómo hacerlo. Y en este punto también, como en tantos otros debates politizados, las soluciones se presentan como parte de extremos opuestos: por un lado el proteccionismo estatal, por otro el libre mercado. A partir de aquí, obviamente, las propuestas varían considerablemente. La mencionada Convención no deja de ser, al respecto, un resultado concreto de este debate a nivel internacional (De Beukelaer y Pyykkönen, 2015).

Antes de continuar es preciso explicitar qué entendemos por diversidad y por industria audiovisual, dos nociones claves. La diversidad es un concepto tiene una larga, rica y bien estudiada trayectoria en tanto principio guía para la elaboración de políticas en el ámbito de las industrias culturales tradicionales, especialmente en relación con los medios de comunicación. Y dado que la diversidad continúa estando presente como uno de los principios centrales en los debates sobre la gobernanza de Internet (Napoli, 2012; Napoli y Karppinen, 2013), es necesario ponerlo sobre la mesa al pensar el audiovisual en un entorno digital. En el caso de la noción de industria audiovisual, es que se defiende que sigue siendo útil pensar "por separado" a los sectores del cine, la radio, la televisión... no como compartimentos estancos pero sí en su especificidad, dado que siguen existiendo aparatos de televisión y salas de cine, y el consumo de medios tradicionales sigue siendo la norma (salvo para los 
millennials). Y, además, porque sigue siendo útil, al decir de García Canclini (2015, p. 122-124), formular a Internet preguntas semejantes a las que hemos estado dirigiendo, por ejemplo, a la televisión: quién dice qué a quién, de qué manera se financia, qué temas favorece, etcétera ${ }^{3}$.

Entendemos por industria audiovisual al conjunto de procesos y actividades, que tienen por objeto la creación, producción, distribución, exhibición o difusión de mercancías con contenido simbólico -sonidos y/o imágenes animadas- que pueden ser consumidas en algún tipo de soporte, a cargo de agentes como empresas o instituciones que participan en las mismas desde un punto de vista económico, político o cultural. Las fases de la industria audiovisual son: creación, producción, distribución, difusión o exhibición y consumo. Sus sectores principales: cine, televisión, radio, música grabada y videojuego.

En lo que refiere a la diversidad de un sistema audiovisual, esta depende de una multiplicidad de factores. A fin de evaluarla deben tenerse en consideración, como mínimo, que:

- La capacidad de producción, distribución y exhibición/emisión de contenidos audiovisuales no esté concentrada en un número reducido de agentes y que estos agentes sean de diferentes tipos de titularidad, tamańo y origen geográfico.

- Los contenidos audiovisuales exhiban diferencias de variedad, balance y disparidad ${ }^{4}$ en términos de valores, identidad y estética. Estos deben reflejar a los múltiples grupos que conviven en una determinada sociedad y hacerse eco de culturas foráneas.

- Los ciudadanos puedan acceder y elegir entre un elevado número de contenidos audiovisuales e, incluso, puedan crearlos y difundirlos.

3 Las definiciones en las que se basa este texto conectan con dos proyectos de $1+D+i$, uno de cuyos resultados fue elaborar un glosario que se encuentra disponible en www.diversidadaudiovisual.org

El proyecto "Diversidad de la industria audiovisual en la era digital" (ref. CSO20 14-52354-R) se desarrolla en el marco del Programa Estatal de I+D+i Orientada a los Retos de la Sociedad del Ministerio de Economía y Competitividad de España. El proyecto "Diversidad cultural y audiovisual: buenas prácticas e indicadores" (ref. CSO20 I-2624I) se desarrolló, durante el trienio 2012-2014, en el marco del Plan Nacional de Investigación Cientifica, Desarrollo e Innovación Tecnológica del mencionado ministerio.

4 La definición de Andrew Stirling $(1998,2007)$ sobre diversidad contempla una combinación de tres componentes: variedad, balance y disparidad. Así, un sistema es más diverso cuanto mayor es el número de categorias que posee, cuanto más equilibradas se encuentran éstas, y cuanto más disímiles son unas de otras. 
Ofrecer un retrato de la industria audiovisual en Internet como primer paso para entender las ventajas y desventajas que existen para su diversidad, supone mapear, cartografiar. Como desarrollamos metodológicamente en otro texto (García Leiva, 2016), mapear implica llevar adelante un ejercicio que tiene por objetivo, además de describir, explicar relaciones (frecuencia, jerarquía, densidad...), analizar el rol que juegan los agentes de peso en un determinado sector y detectar tendencias. Mapear supone representar gráficamente sistemas o estructuras para dar cuenta de sus componentes, ya sean estos actores sociales (individuos, empresas o instituciones) y/o nociones relevantes (como la de concentración empresarial o la de copyright); el lugar que ocupa cada uno de dichos componentes (cuál es su relación jerárquica); y las relaciones existentes entre los mismos (flujos de intercambio).

Dado que tal ejercicio, no exento de problemas, excede por mucho el alcance de estas páginas, se presentan algunas pinceladas de la industria audiovisual en Internet a partir de identificar tipos de agentes así como lógicas en tensión. En relación con los primeros, interesa especialmente describir el alcance que tienen los grupos de comunicación tradicional y los denominados GAFA (Google, Amazon, Facebook y Apple) debido a la intermediación que juegan en la provisión de contenidos audiovisuales. Para ello se parte de la realidad española y se toma como ejemplo el mercado de servicios audiovisuales bajo demanda (VoD), mediado por abono (suscripción o pago por visión, PPV), pero ofrecido a través de la Internet pública y abierta (modalidad más conocida como over the top, OTT) ${ }^{5}$. La oferta online y en abierto, gratuita y bajo demanda (por ejemplo: los servicios de catch-up TV), así como los servicios VoD ofrecidos por un operador de red (por ejemplo: la TV IP), quedan excluidos de la ejemplificación.

5 Según el regulador español, la Comisión Nacional de los Mercados y la Competencia (CNMC, 2015a), los servicios OTT pueden definirse como servicios de todo tipo que se ofertan sobre Internet, sin mediar control alguno ni gestión específica por parte de los operadores de red. Algunos ejemplos son: los juegos online, la distribución de todo tipo de contenidos audiovisuales y de comunicación, los servicios de localización, de intercambio de archivos e información entre personas y/o empresas, de almacenamiento de información $y$, por último, todo tipo de aplicaciones y de software que se distribuyen por Internet. Se contraponen a los servicios IP que prestan y gestionan los operadores usando canales distintos, y, normalmente, con una calidad garantizada. Así, y solo a modo de ejemplo, servicios audiovisuales OTT, como Netflix o YouTube, se difunden a través de la Internet pública abierta, mientras que los servicios de televisión IP, como la oferta de televisión online de un operador de cable o de satélite, se distribuye al cliente final a través de infraestructura gestionada por un operador. En cualquier caso, debe subrayarse que no existe un acuerdo unánime sobre la definición y el alcance de los servicios OTT. 


\section{La industria audiovisual en la era digital}

\section{Lógicas en tensión}

Las lógicas complejas en las que se mueven los agentes que están redefiniendo la cadena de valor de la industria audiovisual deben identificarse y sopesarse con cuidado si se quieren detectar los desafíos y oportunidades para su diversidad en Internet. Entre estas lógicas detectamos las siguientes cinco (Rioux et al., 2015): desmaterialización, desintermediación, descompartimentalización, deslinearización y desterritorialziación.

La desmaterialización implica que a causa del abandono de soportes físicos, es cada vez más difícil distinguir un producto de un servicio, y muchos contenidos culturales que antes eran considerados productos empiezan a ser nombrados como servicios ${ }^{6}$ dado que tendemos no ya a pagar por la propiedad de un bien sino por el acceso a un contenido. Dicho esto, es necesario hacer una reflexión sobre la materialidad de lo virtual: nuestros archivos y mensajes pueden no estar guardados en nuestros dispositivos pero están físicamente alojados en alguna parte (básicamente, servidores que no controlamos).

La desintermediación hace alusión a la capacidad de los consumidores de acceder directamente a la producción de los creadores así como de compartir contenidos en línea. Esto cuestiona sin lugar a dudas el rol de los intermediarios tradicionales. Sin embargo, la desintermediación no se da de forma "pura" sino a través de procesos de re-intermediación y/o desplazamiento (un ejemplo sería la figura del agregador en iTunes).

La descompartimentalización refiere al proceso de convergencia tecnológica y la desaparición de fronteras entre sectores. Los medios de comunicación, las telecomunicaciones y la informática se hermanan en el llamado sector de las tecnologías de la información y la comunicación (TIC),

6 Existe al respecto todo un debate en relación con cómo definir "bienes, servicios y actividades culturales" en el entorno digital. Dado que el concepto de bien parece no tener sentido en el mundo digital, parecería que el término servicios es más apropiado. Sin embargo, no todos los bienes culturales que se digitalizan se transforman, en ausencia de mejor categorización, en un servicio. La cuestión de las etiquetas no es baladí para el comercio internacional, donde las normas que regulan los bienes y servicios pueden tener diferencias fundamentales en el caso de la cultura, ya que los tratados de libre comercio suelen tener obligaciones más estrictas en relación con la liberalización de los bienes que con la de los servicios. Autores como Guèvremont (2013) sugieren que términos como "productos culturales" pueden ser preferibles para el entorno digital. 
y la oferta tiende a converger en un único dispositivo multifunción (la promesa de la black box). Dicho lo cual no debe perderse de vista que la convivencia de estándares y sistemas abiertos, por un lado, y propietarios, por otro - que dan lugar, respectivamente, a redes y códigos abiertos o ecosistemas cerrados (y en buena medida opacos) - no es nada fácil.

Finalmente, la deslinearización permite acceder a un contenido determinado sin estar obligado a seguir el horario de transmisión de la fuente emisora y, por lo tanto, respetar la programación prestablecida. Esta lógica debe ponerse en relación con los fenómenos de prescripción y recomendación que configuran listas de contenidos y propuestas empaquetadas que, con ello, dan lugar a diferentes y nuevas formas editoriales y de programación.

La desterritorialización implica quelas industrias culturales se desenvuelven de manera creciente en un espacio global que trasciende las fronteras del Estado-nación. Los flujos e interacciones ya no pueden comprenderse desde la óptica de las regulaciones y las políticas públicas nacionales. A pesar de esto no hay que olvidar que la producción y consumo cultural en el mundo digital sigue estando vinculada de forma irrenunciable a "territorios de experiencia" basados, por ejemplo, en identidades, lenguas y valores.

\section{Agentes en pugna}

\section{¿Grupos de comunicación us. gigantes globales?}

En España existen cinco grandes grupos privados en el ámbito de las industrias culturales (ver tabla 1), Grupo Prisa, Atresmedia, Unidad Editorial, Mediaset Espańa y Vocento, todos y cada uno de los cuales tiene activos en alguna o todas las fases y sectores de la industria audiovisual. Asimismo operadores de telecomunicaciones como Telefónica, Vodafone u Orange ofrecen también contenidos audiovisuales. Telefónica, por ejemplo, controla la firma DTS cuyo objeto social es comercializar su oferta audiovisual de pago a través de Movistar+. A su vez, como explica el próximo epígrafe, existen numerosas iniciativas en línea que conviven en el mercado español de servicios audiovisuales. 
Tabla I - Grupos privados de la industria cultural española: Algunos indicadores económicos (2016)

\begin{tabular}{lccccc}
\hline & Mediaset & Atresmedia & Prisa & Vocento & Unidad Editorial \\
\hline $\begin{array}{l}\text { Capitalización } \\
\text { bursátil* }\end{array}$ & $3.687,05$ & $2.410,82$ & 454,34 & 162,46 & Sin datos \\
$\begin{array}{l}\text { Ingresos de } \\
\text { explotación** }\end{array}$ & 932,08 & 883,22 & $1.454,7$ & 494,84 & 330 \\
\hline
\end{tabular}

Notas: * En millones de euros hasta el 1/9/2016 según www.bolsamadrid.es.

** En millones de euros según cuenta de resultados consolidada correspondiente al ejercicio anual terminado el 31/12/2015 (en el caso de Unidad Editorial, la fuente es el Informe Anual a 31/12/2015 de su matriz RCS MediaGroup).

Fuente: elaboración propia a partir de información púbica para inversores.

Para magnitudes relacionadas con audiencias televisivas e inversión publicitaria, consultar los informes de la Comisión Nacional de los Mercados y la Competencia. $y$ de las consultoras Infoadex y Barlovento.

En Europa el dibujo es similar al interior de los países: actores dominantes tanto en las redes como en la oferta de contenidos. Existen por un lado poderosos operadores nacionales de telecomunicaciones (como Deutsche Telekom en Alemania, Orange en Francia, BT en Reino Unido y Telecom Italia en Italia) que han acabado por ofrecer contenidos compitiendo por el mercado audiovisual de pago con otros operadores que suelen basar su posición en la explotación de derechos deportivos (Sky, presente en Alemania, el Reino Unido e Italia, o CANALSAT en Francia). A estos hay que sumar los grupos de comunicación tradicionales que, con un modelo de negocio basado en la publicidad, controlan medios de comunicación generalistas (como RTL Group en Alemania, Groupe TF1 en Francia, ITV plc en Reino Unido y Grupo Mediaset en Italia).

Obviamente estos activos deben ponerse en relación con el hecho de que pertenecen a las más importantes corporaciones de medios europeas Bertelsmann AG (Alemania), Vivendi (Francia), BSkyB (Reino Unido) y Fininvest (Italia) - que, a su vez, tienen alcance internacional y se encuentran entre las más grandes del mundo (aunque bien es cierto que los primeros puestos son ocupados por firmas con base en Estados Unidos; ver tabla 2). 
Tabla 2 - Ranking de las mayores corporaciones de medios del mundo (top 20* - 2015)

\begin{tabular}{llcc}
\hline Corporación & Localización & Posición & Ingresos $€$ \\
\hline Comcast & Estados Unidos & 1 & 51,769 \\
Google Inc. & Estados Unidos & 2 & 49,681 \\
The Walt Disney Company & Estados Unidos & 3 & 35,308 \\
News Corp. Ltd./ 2 Ist Century Fox & Estados Unidos & 4 & 30,441 \\
DirecTV, LLC & Estados Unidos & 5 & 24,318 \\
Viacom Inc./ CBS Corp. & Estados Unidos & 6 & 20,767 \\
Time Warner Inc. & Estados Unidos & 7 & 20,594 \\
Sony Entertainment & Japón & 8 & 20,169 \\
Bertelsmann SE E Co. KGaA & Alemania & 9 & 16,675 \\
Apple Inc. & Estados Unidos & 10 & 13,596 \\
Cox Enterprises inc. & Estados Unidos & 11 & 11,972 \\
Liberty Media Corp./ Liberty Interactive & Estados Unidos & 12 & 11,253 \\
Dish Network Corporation & Estados Unidos & 13 & 10,990 \\
Vivendi S.A. & Francia & 14 & 10,089 \\
Tencent & China & 15 & 9,643 \\
Thomson Reuters Corporation & Estados Unidos & 16 & 9,484 \\
Facebook Inc. & Estados Unidos & 17 & 9,384 \\
Rogers Comm. & Canadá & 18 & 8,765 \\
The Hearst Corporation & Estados Unidos & 19 & 7,753 \\
Microsoft Corporation & Estados Unidos & 20 & 7,247 \\
\hline
\end{tabular}

Nota: * Según ingresos obtenidos en el año fiscal 2014 y en miles de millones de euros. Se incluyen compañias que tienen su foco estratégico en la creación de contenidos para prensa, cine, radiotelevisión e Internet (las empresas de cable que poseen infraestructuras se incorporan en tanto producen contenidos). La lista se centra en las compañias que controlan el holding (ej. News Corp.), aunque alguna de sus subsidiarias hubiera registrado mayores ingresos (ej. BSkyB). Fuente: Institut für Medien und Kommunikationspolitik (www.mediadb.eu).

Ahora bien. Lo significativo es que estos agentes, forjados en las redes nacionales de distribución de contenidos audiovisuales (léase: fundamentalmente las redes de difusión herciana, satelital y por cable), han debido adaptarse al entorno digital. Y en este proceso de respuesta a los cambios tecnológicos la adaptación los condujo a desarrollar estrategias de digitalización y distribución multiplataforma, generalmente basadas en la expectativa de 
obtener ventajas en dos frentes (Doyle, 2013). El primero, conseguir una mayor y mejor distribución de los contenidos, porque Internet representa la posibilidad de explotar todo el potencial de lo producido poniéndolo a disposición de múltiples formas y por diferentes vías. El segundo, tener la oportunidad de obtener información sobre los comportamientos y gustos de las audiencias a través del canal de retorno de los dispositivos digitales.

Lo que ocurre es que en esta adaptación estratégica al entorno en línea los grupos de comunicación, que ya tenían que competir con los operadores de telecomunicaciones en la prestación de servicios audiovisuales, deben ahora convivir y competir con otro tipo de actores que son trasnacionales y que no tienen su origen en la industria cultural tradicional o en el ámbito de las telecomunicaciones, y que incluso, algunos, provienen de las industrias del hardware y/o software. Estos actores, conocidos popularmente como GAFA, se erigen como intermediarios clave de los flujos comunicativos en Internet (entre los cuales, obviamente, los contenidos audiovisuales no son la excepción).

Así, la batalla no ha hecho más que empezar, ya que los GAFA tienen ciertas ventajas en lo que a los dos frentes mencionados respecta. Por un lado, la intermediación que ejercen es clave en términos de acceso (buscadores, redes sociales) y distribución (comercialización en línea) porque es global; sin mencionar los intentos más recientes que están haciendo en el ámbito de la producción de contenidos (Miguel y Casado, 2016). Por otro lado, la capacidad que tienen para obtener y gestionar datos de sus usuarios no tiene parangón en la historia de la comunicación social. Es precisamente en esta gestión de lo que se conoce como big data donde reside su ventaja comparativa ya que les permite monetizar el tráfico de los usuarios (Hardy, 2015; Mann, 2014). Los grupos de comunicación, aunque quieran, no tienen la misma capacidad para obtener y explotar esta información.

Otros elementos que contribuyen a su poderío son: un alto grado de financiarización, acompañado de un mayor nivel de capitalización bursátil que los grupos de comunicación (Miguel y Casado, 2016), así como un secretismo casi absoluto sobre su funcionamiento y estrategia.

El caso de Apple es revelador del alcance que pueden tener. Aunque sus dispositivos se fabriquen en Asia, esta multinacional de origen estadounidense presenta un alto grado de integración: comercialización 
de terminales (Macintosh, iPod, iPhone y iPad), sistemas operativos (OS $\mathrm{X}$, iOS), navegador (Safari), programas (Open Office, iWork, iLife...) y contenidos (la tienda virtual iTunes). En marzo de 2016 Apple ocupaba el primer puesto entre las cien mayores empresas del mundo por capitalización bursátil (604.000 millones de dólares estadounidenses). En este ranking, en el que Apple se sitúa por delante de Google (Alphabet Inc), las cinco mayores compañías son estadounidenses y tres proceden del sector tecnológico (ver tabla 3 y PwC, 2016). Hay que consultar hasta los puestos 34 y 40 para que aparezcan las primeras compañías vinculadas con las industrias culturales: los conglomerados de medios y entretenimiento Walt Disney Co y Comcast Corp, respectivamente. El primero con una capitalización bursátil de 162 mil millones de dólares estadounidenses, el segundo con una de 149 mil millones.

Al decir de Badillo (2014, p. 10), el paisaje de la cultura digital tiene su eje principal en un "creciente fértil del Pacífico" que aúna tecnologías, redes y servicios: Asia fabrica los dispositivos a través de los cuales se accede, con software y sistemas operativos estadounidenses, a bienes y servicios culturales producidos mayoritariamente en Estados Unidos. Este acceso está mediado por redes de comunicación interconectadas, que comúnmente conocemos como Internet, cuya infraestructura está en manos de pocas compañías que, también, tienen sede mayoritariamente en Estados Unidos (Level 3, Century Link, AT\&T o Cogent; Ruiz y Barnett, 2015).

$\mathrm{Al}$ no haber pasado por una fase de monopolio nacional, las plataformas de servicios controladas por los GAFA evolucionaron de forma muy distinta a las ofrecidas a través de las redes de comunicación del siglo XX (Conseil National du Numérique, 2014): el reducido nivel de inversión inicial necesario les permitió un rápido crecimiento gracias a una oferta de funciones de uso que propició externalidades de red positivas, ${ }^{7}$ de modo tal que los datos y el

$7 \quad$ La idea es que el valor de una red aumenta conforme se incrementa el número de conexiones, de modo que cuantos más usuarios existan más beneficios reciben todos de la participación (la utilidad de Facebook para conectarse con gente aumenta cuantas más personas son parte de su red). Por lo tanto las externalidades de red son aquellos efectos que hacen que el valor de un producto o servicio para un usuario dependa no sólo de ese producto o servicio sino del número de usuarios que los utilicen. Estos efectos pueden ser, además de positivos, negativos; ello ocurre cuando el número de usuarios atenta contra la utilidad de la red (por ejemplo, cuando el incremento en el tráfico de intercambios genera problemas de congestión, de modo que cada nuevo usuario perjudica a los demás. 
tráfico generado por los usuarios se convirtieron en el eje de la creación de valor. Esta forma de operar convirtió a estos agentes en dominantes en la medida en que su surgimiento y evolución no se han visto contrariados por obstáculos políticos, industriales o financieros (se han caracterizado, por el contrario, por evitar el pago de impuestos y recibir constantes inversiones de capital riesgo; Collin y Colin, 2013).

Tabla 3 - Ranking de conglomerados del mundo por capitalización bursátil (top 20 - 2016*)

\begin{tabular}{|c|c|c|c|c|}
\hline Corporación & Localización & Industria & Posición & Capitalización USD \\
\hline Apple Inc & Estados Unidos & Tecnología & 1 & 604 \\
\hline Alphabet Inc & Estados Unidos & Tecnología & 2 & 518 \\
\hline Microsoft Corp & Estados Unidos & Tecnología & 3 & 437 \\
\hline $\begin{array}{l}\text { Berkshire Hathaway } \\
\text { Inc }\end{array}$ & Estados Unidos & Finanzas & 4 & 350 \\
\hline Exxon Mobil Corp & Estados Unidos & Petróleo y Gas & 5 & 347 \\
\hline Facebook Inc & Estados Unidos & Tecnología & 6 & 325 \\
\hline Johnson E Johnson & Estados Unidos & Sanidad & 7 & 298 \\
\hline General Electric & Estados Unidos & Energía y Electricidad & 8 & 295 \\
\hline Amazon.com & Estados Unidos & Retail & 9 & 280 \\
\hline Wells Farco \& Co & Estados Unidos & Finanzas & 10 & 245 \\
\hline ATET Inc & Estados Unidos & Telecomunicaciones & 11 & 241 \\
\hline Nestle SA & Suiza & Bienes de consumo & 12 & 239 \\
\hline China Mobile Ltd & Hong Kong & Telecomunicaciones & 13 & 228 \\
\hline ICBC Ltd & China & Finanzas & 14 & 228 \\
\hline Procter E Gamble Co & Estados Unidos & Bienes de consumo & 15 & 223 \\
\hline $\begin{array}{l}\text { Verizon } \\
\text { Communications Inc }\end{array}$ & Estados Unidos & Telecomunicaciones & 16 & 221 \\
\hline $\begin{array}{l}\text { JPMorgan Chase } \\
\text { E Co }\end{array}$ & Estados Unidos & Finanzas & 17 & 217 \\
\hline Wal-Mart Stores Inc & Estados Unidos & Retail & 18 & 215 \\
\hline Roche Holding AG & Suiza & Sanidad & 19 & 214 \\
\hline PetroChina Co Ltd & China & Petróleo y Gas & 20 & 205 \\
\hline
\end{tabular}

Nota: * Capitalización bursátil a 31/03/2016 y en miles de millones de dólares estadounidenses.

Fuente: PwC (20/6). 
La existencia de un audiovisual en línea diverso pues no está asegurada, ya que la fórmula de éxito de estos agentes se basa en modelos cerrados de comercialización de contenidos que añaden también restricciones a la reproducción y circulación por parte de y entre los usuarios. La noción de externalidades de red permite entender por qué, además de globales, son poderosos: si iTunes controla aproximadamente el $70 \%$ del mercado de descargas de música y Google el 70\% de las búsquedas, Facebook centraliza más de la mitad del tráfico en redes sociales (Hardy, 2015: 127).

Como ilustra la tabla 4, el ranking de webs más consultadas desde ordenadores en los países más ricos del mundo pone de manifiesto con contundencia el papel de los GAFA como puerta de acceso y escaparate para los contenidos online. El liderazgo de los sitios web propiedad de Google es absoluto, así como también es evidente la popularidad de las redes sociales, con Facebook presente en los cinco primeros puestos en todos los países. También debe señalarse que, junto con los sitios de la Fundación Wikimedia (que incluyen la muy conocida Wikipedia), entre las webs más visitadas en cada país figuran sitios nacionales. Salvo excepciones, como la $\mathrm{BBC}$ en el Reino Unido, la mayoría son propiedad de grandes grupos de comunicación (como el Grupo Prisa en España o News Corp en Australia). 
Tabla 4 - Top 10 de las web properties más consultadas desde ordenadores, 2015

\begin{tabular}{|c|c|c|c|c|c|c|c|c|}
\hline & Reino Unido & Francia & Alemania & Italia & $\begin{array}{l}\text { Estados } \\
\text { Unidos }\end{array}$ & Japón & Australia & España \\
\hline I & Sitios Google & Sitios Google & Sitios Google & Sitios Google & Sitios Google & Sitios Google & Sitios Google & Sitios Google \\
\hline 2 & $\begin{array}{c}\text { Sitios } \\
\text { Microsoft }\end{array}$ & Facebook & Facebook & Facebook & Facebook & Sitios Yahoo & $\begin{array}{c}\text { Sitios } \\
\text { Microsoft }\end{array}$ & Facebook \\
\hline 3 & Facebook & $\begin{array}{c}\text { Sitios } \\
\text { Microsoft }\end{array}$ & Sitios Microsoft & Italia Online & Sitios Yahoo & Sitios Microsoft & Facebook & $\begin{array}{c}\text { Sitios } \\
\text { Microsoft }\end{array}$ \\
\hline 4 & $\begin{array}{c}\text { Sitios } \\
\text { Amazon }\end{array}$ & $\begin{array}{c}\text { CCM- } \\
\text { Benchmark }\end{array}$ & $\begin{array}{c}\text { Sitios } \\
\text { Amazon }\end{array}$ & $\begin{array}{c}\text { Sitios } \\
\text { Microsoft }\end{array}$ & Sitios Microsoft & FC2 Inc. & Sitios Yahoo & $\begin{array}{l}\text { Unidad } \\
\text { Medios } \\
\text { Digitales }\end{array}$ \\
\hline 5 & Sitios BBC & Webedia Sites & eBay & Banzai & $\begin{array}{c}\text { Sitios } \\
\text { Amazon }\end{array}$ & $\begin{array}{c}\text { LINE } \\
\text { Corporation }\end{array}$ & $\begin{array}{l}\text { News Corp } \\
\text { Australia }\end{array}$ & Grupo Prisa \\
\hline 6 & eBay & Sitios Yahoo & $\begin{array}{c}\text { Axel Springer } \\
\text { SE }\end{array}$ & $\begin{array}{l}\text { Sitios } \\
\text { Wikimedia } \\
\text { Foundation }\end{array}$ & AOL Inc. & DMM & $\begin{array}{l}\text { TripAdvisor } \\
\text { Inc. }\end{array}$ & Sitios Yahoo \\
\hline 7 & Sitios Yahoo & Orange Sites & $\begin{array}{l}\text { Deutsche } \\
\text { Telekom }\end{array}$ & $\begin{array}{c}\text { Sitios } \\
\text { Amazon }\end{array}$ & Mode Media & $\begin{array}{c}\text { Sitios } \\
\text { Amazon }\end{array}$ & $\begin{array}{c}\text { Sitios } \\
\text { Wikimedia } \\
\text { Foundation }\end{array}$ & Vocento \\
\hline 8 & $\begin{array}{c}\text { Sitios } \\
\text { Wikimedia } \\
\text { Foundation }\end{array}$ & $\begin{array}{c}\text { Schibsted } \\
\text { Media Group }\end{array}$ & $\begin{array}{l}\text { Hubert Burda } \\
\text { Media }\end{array}$ & eBay & $\begin{array}{c}\text { Sitios } \\
\text { Wikimedia } \\
\text { Foundation }\end{array}$ & Rakuten Inc. & eBay & $\begin{array}{c}\text { Schibsted } \\
\text { Media Group }\end{array}$ \\
\hline 9 & $\begin{array}{l}\text { TripAdvisor } \\
\text { Inc. }\end{array}$ & $\begin{array}{l}\text { Sitios } \\
\text { Amazon }\end{array}$ & $\begin{array}{l}\text { United-Internet } \\
\text { Sites }\end{array}$ & Sitios Yahoo & CBS Interactive & Facebook & Mode Media & $\begin{array}{c}\text { Sitios } \\
\text { Amazon }\end{array}$ \\
\hline 10 & Apple Inc. & Solocal Group & $\begin{array}{c}\text { Sitios } \\
\text { Wikimedia } \\
\text { Foundation }\end{array}$ & $\begin{array}{l}\text { Gruppo } \\
\text { Editoriale } \\
\text { Espresso }\end{array}$ & eBay & $\begin{array}{l}\text { Kadokawa } \\
\text { Dwango } \\
\text { Corporation }\end{array}$ & $\begin{array}{c}\text { Telstra } \\
\text { Corporation } \\
\text { Ltd. }\end{array}$ & $\begin{array}{l}\text { Alibaba.com } \\
\text { Corporation }\end{array}$ \\
\hline
\end{tabular}




\section{El caso del VoD de pago OTT}

Partiendo de lo explicado, detenerse en el caso del mercado de pago de VoD OTT puede ayudar a esclarecer el lugar que ocupa cada uno de estos tipos de agentes en el escenario de la industria audiovisual en línea. Si a la descripción de ofertas y modelos de negocio que sigue, ańadimos información de contexto sobre las magnitudes del mercado del VoD, podemos sugerir la hipótesis de que, a diferencia de lo que ocurre con la televisión tradicional (consumida fundamentalmente en directo), dominada en cada mercado por agentes nacionales y/o continentales, el VoD de pago OTT se está configurando como un mercado controlado por agentes de alcance trasnacional y origen estadounidense. Ya que si bien existen, como veremos, iniciativas nacionales, la presencia y poder de actores como Netflix parece ir en aumento.

Desde el punto de vista del consumidor español de $\mathrm{VoD}$ de pago OTT, lo primero que debe señalarse es la oferta de los grupos españoles de comunicación (Mediaset y Atresmedia), a la que se suman iniciativas de origen tanto nacional como foráneo (sintetizadas en la tabla 5), aunque con importancia diferente. Dentro del primer grupo encontramos a Filmin, Filmotech, Feelmakers, Cineclick, ADNstream y Televeo (por el camino se han quedado lanzamientos como 400 Films).

Entre las plataformas originadas en España, por lo tanto, cabe distinguir tres grandes tipos: a) aquellas derivadas del negocio televisivo de grandes grupos de comunicación (Mitele y Atresplayer); b) aquellas surgidas de la colaboración entre productoras y distribuidoras nacionales (Filmin, Filmotech y Cineclick, para el caso de las empresas más consolidadas; Feelmakers, para el caso de agentes pequeños y medianos); y c) aquellas que pertenecen a emprendimientos comerciales de escala pequeña (ADNstream y Televeo). El segundo de estos tres subgrupos destaca por su especialización en la oferta, con estrategias de posicionamiento claras por segmentos de público, ya que el primero presenta una clara orientación a la explotación de títulos previamente adquiridos y/o producidos, y el tercero supone casos irrelevantes desde el punto del vista del negocio y variopintos desde la óptica de los contenidos ofrecidos. 
Tabla 5 - España: mercado de VoD de pago OTT, 2016

\begin{tabular}{|c|c|c|c|c|c|}
\hline \multirow[t]{2}{*}{ Plataforma } & \multirow[t]{2}{*}{ Propietario } & \multicolumn{2}{|c|}{ Oferta } & \multicolumn{2}{|c|}{$\begin{array}{c}\text { Modelo negocio (tarifa } \\
\text { mínima/€) }\end{array}$} \\
\hline & & Cine & Series & Abono/mes & PPV \\
\hline \multicolumn{6}{|c|}{ Origen español } \\
\hline Mitele & Mediaset & 42 & 60 & - & 1.21 \\
\hline Atresplayer & Atresmedia & \multicolumn{2}{|c|}{350} & - & 0.99 \\
\hline Filmin & $\begin{array}{l}\text { Comunidad } \\
\text { Filmin }\end{array}$ & \multicolumn{2}{|c|}{8.500} & 8 & 1.95 \\
\hline Filmotech & EGEDA & 2.148 & & 6.95 & 0.50 \\
\hline Cineclick & Cineclick & 1566 & - & 4.99 & 0.99 \\
\hline Feelmakers* & Content Line & \multicolumn{2}{|c|}{700} & 6.00 & 0.90 \\
\hline ADNstream & $\begin{array}{l}\text { Fractal Internet } \\
\text { Consultancy }\end{array}$ & \multicolumn{2}{|c|}{160} & 2.99 & 1.50 \\
\hline Televeo & $\begin{array}{c}\text { M4V Interactive } \\
\text { Business }\end{array}$ & \multicolumn{2}{|c|}{192} & - & 1.00 \\
\hline \multicolumn{6}{|c|}{ Origen foráneo } \\
\hline $\begin{array}{l}\text { Google Play } \\
\text { Movies }\end{array}$ & Google & \multicolumn{2}{|c|}{ información no disponible } & - & 1.99 \\
\hline iTunes Videos & Apple & $45.000^{* *}$ & $\begin{array}{l}85.000 * * \\
\text { episodios }\end{array}$ & - & 1.99 \\
\hline Netflix & Netflix & 1569 & 356 títulos & 7.99 & - \\
\hline Waki TV & Rakuten & 5.000 & 85 & 6.99 & 1.99 \\
\hline $\begin{array}{l}\text { Vimeo On } \\
\text { Demand }\end{array}$ & IAC & \multicolumn{2}{|c|}{$2.000^{* *}$} & - & 1.00 \\
\hline MUBI & $\begin{array}{c}\text { Varios } \\
\text { accionistas } \\
\text { (Efe Cakarel } \\
\text { fundador) }\end{array}$ & \multicolumn{2}{|l|}{3.328} & 5.99 & - \\
\hline ITV Essentials & ITV plc & \multicolumn{2}{|c|}{82} & 3.99 & - \\
\hline Africafilms & Soon & \multicolumn{2}{|c|}{625} & - & 2.00 \\
\hline
\end{tabular}

Notas: * Especializada en documentales, animación y cortometrajes.

** Catálogo global; sin datos para España.

Fuente: elaboración propia a partir de la base de datos MAVISE

e información de las plataformas. 
Dentro del grupo de iniciativas foráneas, la oferta estaba conformada, a octubre de 2016, por plataformas originadas en otro país, las más importantes de las cuales tienen base en Estados Unidos y desembarcaron en España en el contexto de su estrategia de expansión europea. En este grupo, como en el anterior, cabe distinguir distintos tipos de iniciativas que pueden organizarse también en tres subgrupos: a) las de alcance global, ya sea vinculadas a alguno de los GAFA (Google Play y iTunes Video ${ }^{8}$ ) o a compañías multinacionales (Netflix y Waki, de Rakuten, el "Amazon japonés"); b) las internacionales, cuya presencia geográfica depende de acuerdos estratégicos con partners regionales (Vimeo, que es propiedad de un conglomerado de medios de origen estadounidense, MUBI e ITV Essentials); y c) las que pertenecen a emprendimientos comerciales de menor talla, lanzadas en colaboración (Africafilms).

$\mathrm{Si}$ en este último caso se trata de propuestas de nicho (Africafilms nació de tres productoras y distribuidoras africanas y su catálogo se dedica a películas africanas o sobre África), y el primero de los subgrupos ofrece básicamente audiovisual mainstraim, el segundo es el que agrupa estrategias más segmentadas (siendo peculiar el caso de ITV Essentials por consistir básicamente en la puesta a disposición de la oferta de un operador de televisión británico en trece países europeos).

Dado que es muy difícil encontrar cifras de ingresos de estos emprendimientos empresariales, muchas veces camufladas en el conjunto de las operaciones audiovisuales y/o en línea de las plataformas, la consulta de diversos informes sectoriales puede al menos dar pistas del orden de magnitudes en juego (aunque no todos los datos refieren sólo al VoD OTT).

En España, en el año 2014, los ingresos por pago por visión y vídeo bajo demanda registraron un avance interanual del 15,2\%, hasta los 24,6 millones de euros. De estos, 19,2 millones correspondieron al alquiler de películas y el resto, 5,4 millones, a otros acontecimientos que incluyen el alquiler de conciertos, de series televisivas, documentales y eventos deportivos (CNMC, 2015b). Esto debe ponerse en relación con la caracterización efectuada por el regulador sobre los servicios OTT en España (CNMC, 2015a), que indica que en 2014 , a pesar de que el $36,7 \%$ de los individuos con acceso a Internet

8 A finales de 2016 se espera el desembarco inminente de Amazon Instant Video en España, Francia e Italia (hasta entonces disponible en el Reino Unido y Alemania), así como de HBO Now. 
visionaban contenidos audiovisuales sobre Internet con una relativa frecuencia, el porcentaje de hogares que los contrataba era muy reducido: un 0,6\%. La conclusión presentada era que estos servicios de pago, rivales a la televisión por abono ofertada por los operadores, no eran todavía significativos. Esto también era puesto en relación con el hecho de que la televisión de pago podría estar limitada por una oferta gratuita significativa, tanto en Internet como en TDT.

Cifras más recientes demostrarían el aumento sostenido en el gasto dedicado a consumir video OTT (International Video Federation, 2016): 72.4 millones de euros en 2015 (ver tabla 6; debe hacerse notar que el cómputo incluye también la venta de películas y series).

Tabla 6 - España: gasto dedicado al consumo de video OTT, 2009-2015 (en millones de euros)*

\begin{tabular}{ccccccc}
\hline $\mathbf{2 0 0 9}$ & $\mathbf{2 0 1 0}$ & $\mathbf{2 0 1 1}$ & $\mathbf{2 0 1 2}$ & $\mathbf{2 0 1 3}$ & $\mathbf{2 0 1 4}$ & $\mathbf{2 0 1 5}$ \\
\hline 0.9 & 2.1 & 6.3 & 14.4 & 26.8 & 44.0 & 72.4 \\
\hline
\end{tabular}

Nota: * Se incluyen tanto la compra como el alquiler de películas y series

distribuidas OTT, mediando algún tipo de transacción o suscripción.

Se excluyen los eventos en directo, el contenido para adultos y los deportes.

Fuente: IVF (2016).

En Europa se detecta esta misma tendencia: si en 2009 más de tres cuartas partes del consumo audiovisual bajo demanda era gestionado por operadores de redes cerradas (servicios de televisión de pago, redes de cable), y menos de un cuarto tenía lugar en la Internet abierta, es decir, OTT, en 2012 el segundo tipo sobrepasó al primero en un movimiento que no ha dejado de ascender: el gasto dedicado a consumir VoD OTT (gratuito y de pago) creció, entre 2009 y 2013, al menos un 57,9\% anual hasta alcanzar un total de 100,8\% (Grece et al., 2015). Ya en 2013, en el conjunto de la oferta de VoD, los servicios mediados por suscripción y alquiler superaban a los consumidos gratuitamente o por adquisición (ver tabla 7).

Tabla 7 - Europa: modelos de negocio VoD, 2013 (\% share)

\begin{tabular}{cccc}
\hline TV VoD & Suscripción & Alquiler & Venta \\
\hline 39,95 & 26,43 & 14,59 & 19,03 \\
\hline
\end{tabular}

Fuente: IHS/ IVF (en Grece et al., 20/5). 
Como bien explica el último de los informes del Observatorio Audiovisual Europeo sobre $\mathrm{VoD}$ ( $o p$. cit.), esta tasa de crecimiento debe ponerse en relación con el hecho de que operadores OTT como Netflix o Waki.tv han entrado en más mercados europeos a partir de 2014, y con que los agentes tradicionales, como Canal+, SBSkyB, o Sky Deutschland, han también lanzado sus ofertas OTT diferenciadas (respectivamente, Canal Play, Now TV, Sky Snap). Evidentemente, esta expansión en la oferta ha sido acompañada por cambios en la demanda: la penetración de banda ancha en los hogares europeos, así como la adquisición de terminales conectados, no ha dejado de crecer $^{9}$. Se estima que el hecho de que el video ofrecido OTT pueda reproducirse sin grandes dificultades en la pantalla del televisor, además de ser consumido en dispositivos móviles, impulsará aún más el consumo europeo de VoD OTT.

Dicho esto, conviene no olvidar que los mercados europeos presentan una distribución muy desigual en la adopción y consumo de servicios VoD: el Reino Unido y los países nórdicos (Suecia, Dinamarca, Finlandia y Noruega) efectuaban, en 2013, casi tres cuartas partes del total del gasto europeo en VoD por suscripción. El Observatorio Audiovisual Europeo nos recuerda que los mercados maduran a velocidades diferentes; de ahí que las predicciones de consultoras como Digital TV Research, a partir del desembarco de Netflix en seis países europeos en septiembre de 2014, y el anuncio de la inminente llegada de Amazon Instant Video, deban matizarse. Según esta consultora ${ }^{10}$, aproximadamente el 7\% de los hogares con televisión de Europa Oriental se suscribirán a servicios VoD para 2020, mientras la penetración alcanzará a casi el 30\% en Europa Occidental.

En la actualidad, según un informe de la Unión Europea de Radiodifusión ${ }^{11}$ casi el 11\% de los hogares europeos tiene una suscripción a servicios de VoD (la mayoría de los cuales están en el Reino Unido, Holanda, Irlanda y los países nórdicos) y el líder indiscutible de este segmento es Netflix (con el 52\% del mercado europeo). Sin embargo, el impacto de este tipo de servicio en el

9 Aunque exceda los objetivos de esta descripción, ha de hacerse notar la importancia de los aqui denominados grandes agentes globales en la configuración del equipamiento conectado (desde televisores hasta adaptadores y aplicaciones): Apple TV, Amazon Fire TV, Roku media player, Google's Chromecast, Amazon's Fire TV Stick, Mozilla's Matchstick, Microsoft's Wireless Display Adapter...

10 http://www.homemediamagazine.com/streaming/report-20-european-tv-homes-have-svod-2020-34036

$1 /$ https://www.ebu.ch/news/2016/06/european-svod-reach-50-mil-2020 
conjunto del consumo audiovisual es todavía modesto. En el Reino Unido, por ejemplo, el visionado de VoD por suscripción representa sólo el 4\% (11 minutos) del total diario de consumo audiovisual.

A nivel global, IDATE (2015) estima que en el mundo había 84,5 millones de suscripciones a servicios VoD en 2014, la mayor parte de las cuales se encontraban en América del Norte, especialmente en Estados Unidos. Este país es por lejos el mercado más grande del mundo, en cuyo interior Netflix, Amazon Prime Instant Video e iTunes se encuentran entre los actores más importantes. Y como estos han decidido expandirse al mundo entero, lo que se está configurando para el caso de los servicios audiovisuales de pago bajo demanda, ofrecidos vía OTT, es un oligopolio global. A falta de datos de Google y Amazon, es pertinente señalar que el servicio de alquiler de VoD de iTunes está disponible en unos 110 países (en muchos de los cuales Apple compite con agentes de origen nacional como Orange en Francia, maxdome en Alemania o Videoland en Holanda), y que Netflix dominaba en 2014 el mercado mundial de $\mathrm{VoD}$ por suscripción, ofrecido vía OTT, detentando aproximadamente el 66\% del mismo (IDATE, 2015, p. 74).

Según la consultora Research and Markets ${ }^{12}$, Netlix tendrá casi 115 millones de suscriptores para 2020 (50,4 millones en Estados Unidos), y el total de ingresos para el conjunto de operadores de todos los tipos de oferta de VoD por suscripción, en 200 países, ascenderá a 26.784 millones de dólares estadounidenses para la misma fecha (diez veces más que los 2.644 millones registrados en 2010).

\section{El audiovisual en Internet: desafíos y oportunidades}

En definitiva, y en función del retrato hasta aquí presentado, es posible apuntar un conjunto de ventajas y desventajas para la diversidad de la producción, distribución/exhibición y consumo de audiovisual en Internet. Como se señaló al comienzo del texto, Internet presenta tanto oportunidades como riesgos.

En el conjunto de las oportunidades para la diversidad sobresalen las siguientes:

12 https://globenewswire.com/news-release/2015//2/10/794425/0/en/Global-SVOD-Subscription-Video-onDemand-Forecasts-2015-2020-SVOD-revenues-for-all-operators-in-200-countries-will-reach-2-67-Billion.html 
- Producción

- Impulso a la experimentación e innovación, además de mayor flexibilidad, a partir del uso de nuevas herramientas.

- Mayor competitividad, debido a la desaparición/reducción de costes de producción.

- Aparición de nuevas ventanas de promoción y oportunidades de financiación, así como de alternativas para la autogestión de la carrera profesional.

- Contacto directo con el público.

- Distribución/ Exhibición

- Mayor competitividad, debido a la desaparición/reducción de costes.

- Generación de nuevos mercados, nichos y modelos de negocio.

- Más facilidad y rapidez en la comunicación con los consumidores.

- Consumo

- Vasta oferta a disposición.

- Aumento y mejora, en términos de facilidad, rapidez, movilidad y coste, del acceso a bienes y servicios audiovisuales.

- Más ocasiones para la participación en la vida cultural y el diálogo intercultural.

- Posibilidad de iniciarse/desarrollarse en el mundo de la creación y la colaboración cultural en paralelo a su consumo.

En lo que respecta a los riesgos debemos señalar:

- Producción

- Más oportunidades para usos no autorizados de la creación cultural.

- Aumento de la competencia que no proviene necesariamente de un único ámbito geográfico o sectorial y que puede tener origen incluso en los contenidos generados por los usuarios. 
- Necesidad de enfrentar nuevas re-intermediaciones no siempre ejercidas por los agentes ya conocidos y, por tanto, debilitamiento de la capacidad de negociación.

- Dificultades para monetizar bienes y servicios dada la enorme competencia por los mismos recursos (pago y publicidad) y los problemas/cuestionamientos que enfrentan los ingresos por copyright.

- Distribución/ Exhibición

- Reconfiguración de la cadena de valor a partir de la transformación de procesos y roles, lo cual cuestiona la existencia o desempeño de los actores existentes (aparición nuevos intermediarios).

- Si hace ya una década se hablaba de "Hollyweb" (Frau-Meigs, 2011: 196), como la alianza de Hollywood con las industrias web, que funciona como un modelo de provisión de información, la tendencia a la concentración es hoy, además, muy clara: se refuerzan posiciones ya dominantes (los grupos de comunicación, las majors) a la vez que se consolidan los llamados GAFA. El ejemplo aquí utilizado del $\mathrm{VoD}$ de pago OTT es, al respecto, revelador.

- Consumo

- Brechas en el acceso debido a distintos tipos de disparidades o desigualdades (geográficas, etarias, de renta o alfabetización...)

- Peligro de que las recomendaciones, la publicidad y las búsquedas personalizadas acaben orientando el acceso de modo que se construyan "bucles de conocimiento" que limiten los descubrimientos.

- Sobreoferta o mayor visibilidad para cierto tipo de contenidos (comerciales) que se presentan, además, de forma dominante en una lengua (inglés). 


\section{A modo de conclusión: barreras para la diversidad del audiovisual en línea}

En este escenario, entonces, si tuviéramos que elaborar una relación de elementos que dificultan el fortalecimiento de la diversidad de la industria audiovisual en el entorno digital, las barreras serían tecnológicas, económicas, sociales, legales y políticas.

En el plano tecnológico, la falta de infraestructuras y herramientas adecuadas y/o asequibles pueden afectar tanto a la producción (por ejemplo impidiendo el desarrollo creativo de las geografías con menor renta per cápita) como al consumo audiovisual (por ejemplo impidiendo un acceso de calidad a las producciones culturales en zonas rurales). Los últimos datos disponibles de la UIT (2015) reflejan, por poner el caso, la distancia enorme que hay entre países si miramos los indicadores de penetración de banda ancha. Esto, obviamente, debe también considerarse en relación con aspectos sociales como la ausencia de alfabetización digital o know how suficiente para usar y aprovechar las nuevas redes y tecnologías.

Desde un punto de vista económico, se verifica la consolidación de las economías de escala y gama de los agentes ya dominantes en el entorno no digital. Muchas de las características de las industrias culturales tradicionales (costes altos de producción pero costes marginales bajos de reproducción y distribución) se conservan en el entorno digital. De forma tal que aunque existan ocasiones para que nuevos jugadores desafíen a los existentes, allí donde los costes de creación siguen siendo elevados (como por ejemplo en la producción de un largometraje) las barreras siguen en pie. Internet profundiza esta cuestión porque provee beneficios de escala y gama a los agentes ya importantes: los avances tecnológicos no han acabado con los monopolios "naturales" de las economías de escala allí donde existen (Hardy, 2014), por lo que únicamente quienes aúnan saber y poder sobre capital y tecnología en Internet (los GAFA), a escala trasnacional, son los que han podido cuestionar en el ámbito audiovisual el papel de las industrias tradicionales.

En lo que refiere al ámbito legal, se detectan numerosos vacíos normativos o inadecuación de normas. Por ejemplo, en relación con la propiedad intelectual, la privacidad, la publicidad, los derechos de rectificación y réplica 
o la tributación fiscal. Al respecto puede citarse el ejemplo de lo que algunos analistas (Collin y Colin, 2013) llaman "el doble sándwich holandés-irlandés", que se preparan las corporaciones con base en Estados Unidos, con "relleno" de algún paraíso fiscal (Gibraltar, Bermudas o Islas Caimán), para implementar una estrategia de ingeniera fiscal que suponga jurisdicciones diferentes para producir, gestionar los derechos de explotación y tributar.

No debemos olvidar, finalmente, que existen en muchos países importantes barreras políticas a superar, como la presencia de censura o controles políticoideológicos que pueden eliminar una parte de la oferta cultural y/o condicionar el acceso a la misma. Aquí, además de pensar en los casos más sonados, como puede ser el ejemplo de Google en China, hay que considerar los que por anecdóticos no dejan de ser elocuentes e inquietantes: en abril de 2015 el Ministerio de Cultura de Brasil publicó en su Facebook una foto de una pareja de indios Botocudos de Espíritu Santo para promocionar un acervo de fotografías históricas digitalizadas; la empresa la retiró alegando normas globales pero la restituyó rápidamente ante el revuelo y cuando el gobierno anunció que iniciaría acciones legales por lo que consideraba un acto de censura.

\section{Bibliografía}

ALBORNOZ, L. A. y GARCÍA LEIVA, M.T. Diversidad cultural, industria audiovisual y gobernanza. In: ALBORNOZ, L. A. y GARCÍA LEIVA, M.T. (eds.). Diversidad e industria audiovisual: el desafío cultural del siglo XXI. México D.F.: FCE, 2017.

BADILLO, A. ¿̨Fuera de juego global?: los grupos culturales espańoles y europeos en las redes mundiales. ARI No 22. Madrid: RIE, 2014.

CNMC. Caracterización del uso de algunos servicios over the top en Espańa (comunicaciones electrónicas y servicios audiovisuales). Documento de Trabajo no 4, 2015a. Disponible en: https://www.cnmc.es/Portals/0/Ficheros/Telecomunicaciones/ Informes/20150130_DOC_OTT_21_11_CC_REVISADO.pdf

CNMC. Informe Económico Sectorial de las Telecomunicaciones y el Audiovisual 2015. 2015b. Disponible en: https://www.cnmc.es/es-es/telecomunicacionesysaudiovisuales/ informestelecomunicacionesysaudiovisual/informesanuales.aspx

COLLIN, P.; COLIN, N. Task Force on Taxation of the Digital Economy. Report to the Minister for the Economy and Finance, the Minister for Industrial Recovery, the Minister 
Delegate for the Budget and the Minister Delegate for Small and Medium-Sized Enterprises, Innovation and the Digital Economy. París: Ministère de l'économie et des finances/ Ministère du redressement productif, 2013.

CONSEIL NATIONAL DU NUMÉRIQUE. Neutralidad de las plataformas. Fomentar un entorno digital abierto y sostenible. Dictamen no 2014-2 del Consejo Nacional Digital sobre la neutralidad de las plataformas. París: Ministère de l'économie du redressement productif et du numérique.

DE BEUKELAER, C.; PYYKKÖNEN, M.; SINGH, J. P. (eds.). Globalization, Culture and Development: the UNESCO Convention on Cultural Diversity. Hampshire y New York: Palgrave Macmillan, 2015.

DE BEUKELAER, C.; PYYKKÖNEN, M. Introduction: UNESCO’s "Diversity Convention" - Ten Years on. In: DE BEUKELAER, C., PYYKKÖNEN, M. y SINGH, J. P. (eds.). Globalization, Culture and Development: the UNESCO Convention on Cultural Diversity. Hampshire y New York: Palgrave Macmillan, 2015.

DOYLE, G. Understanding media economics. Second edition. Londres: SAGE, 2013.

FRAU-MEIGS, D. Media Matters in the Cultural Contradictions of the "information society" - Towards a human-rights-based governance. Estrasburgo: Consejo de Europa, 2011.

FREEDMAN, D. Web 2.0 and the death of the blockbuster economy. In: CURRAN, J., FENTON, N. y FREEDMAN, D. Misunderstanding the Internet. Londres y Nueva York: Routledge, 2012.

GARCÍA CANCLINI, N. El mundo entero como lugar extraño. Barcelona: Gedisa, 2015.

GARCÍA LEIVA, M. T. ¿Cómo mapear la diversidad de la industria audiovisual en la era digital? Notas metodológicas. CIC. Cuadernos de Información y Comunicación, n. 21, p. 13-25, 2016.

GRECE, C.; LANGE, A.; SCHNEEBERGER, A.; VALAIS, S. The development of the European market for on-demand audiovisual services. Estrasburgo: European Audiovisual Observatory, 2015.

GUĖVREMENONT, V. Preliminary reflection on the implementation of the Convention on the Protection and Promotion of the Diversity of Cultural Expressions in the digital age. Informe elevado a la $4^{\mathrm{a}}$ Conferencia de las Partes de la Convención sobre la Protección y Promoción de la Diversidad de las Expresiones Culturales (UNESCO, París, junio de 2013). 
Disponible en: http://papers.ssrn.com/sol3/papers.cfm?abstract_id=2490278 [consulta: $10 / 01 / 2015]$.

HARDY, J. Critical Political Economy of the Media. An Introduction. Oxon \& Nueva York: Routledge, 2014.

IDATE. Digiworld Yearbook 2015. Montpellier: IDATE, 2015.

INTERNATIONAL VIDEO FEDERATION. Video market: Spain, 2016. Disponbile en: http://www.ivf-video.org/new/public/media/2016_IVF_Spain.pdf

MANN, D. Welcome to the unregulated wild, wild, digital west. Media Industries, v. 1, n. 2, 2014. Disponible en: http://www.mediaindustriesjournal.org/index.php/mij/article/view/47

MIGUEL DE BUSTOS, J. C.; CASADO, M. A. Emergencia de los GAFA y cambios en el sistema comunicativo global. Telos, n 104, junio-septiembre, p. 38-47, 2016.

NAPOLI, P. Diminished, enduring, and emergent diversity policy concerns in an evolving media environment. In: PAGER, S. y CANDEUB, A. (org.). Transnational Culture in the Internet Age. Glos y Massachusetts: Edward Elgar, 2012.

NAPOLI, P.; KARPPINEN, K. Translating diversity to Internet governance. First Monday, v. 8, n. $12,2013$.

OFCOM. International Communications Market Report. Londres: Ofcom, 2015. Disponible en: http://stakeholders.ofcom.org.uk/binaries/research/cmr/cmr15/icmr15/ icmr_6.pdf [consulta: 2/9/2016].

PAGER, S.; CANDEUB, A. Introduction: navigating in the dark when bits have no borders. In: PAGER, S. y CANDEUB, A. (org.). Transnational Culture in the Internet Age. Glos y Massachusetts: Edward Elgar, 2012.

PwC. Global Top 100 Companies by Market Capitalisation, 2016. Disponible en: https://www.pwc.com/gr/en/publications/assets/global-top-100-companies-by-marketcapitalisation.pdf [consulta: 2/9/2016].

RIOUX, M. et al. For a diversified Networked Culture. Bringing the Convention on the Protection and Promotion of the Diversity of Cultural Expressions (CPPDCE) in the digital age. Montreal: CEIM, 2015. Disponible en: http://www.ieim.uqam.ca/IMG/pdf/ rapportcdec-court-en-vfinale.pdf [consulta: 14/10/2015].

RUIZ, J.; BARNETT, G. Who owns the international internet networks? The Journal of International Communication, v. 21, n. 1, p. 38-57, 2015. 
UIT. The State of Broadband 2015. Ginebra: UIT \& UNESCO, 2015. Disponible en: http://www.broadbandcommission.org/documents/reports/bb-annualreport2015.pdf [consulta: 1/9/2016].

ZALLO, R. Estructuras de la comunicación y de la cultura. Políticas para la era digital. Barcelona: Gedisa, 2011.

\section{Challenges and opportunities for audiovisual diversity in the Internet}

\section{Abstract}

At the gates of the first quarter of the XXI century, nobody doubts the fact that the value chain of the audiovisual industry has suffered important transformations. The digital era presents opportunities for cultural enrichment as well as displays new challenges. After presenting a general portray of the audiovisual industries in the digital era, taking as a point of departure the Spanish case and paying attention to players and logics in tension, this paper will present some notes about the advantages and disadvantages that exist for the diversity of audiovisual production, distribution and consumption online. It is here sustained that the diversity of the audiovisual sector online is not guaranteed because the formula that has made some players successful and powerful is based on walled-garden models to monetize contents (which, besides, add restrictions to their reproduction and circulation by and among consumers). The final objective is to present some ideas about the elements that prevent the strengthening of the diversity of the audiovisual industry in the digital scenario. Barriers to overcome are classified as technological, financial, social, legal and political.

Keywords: Audiovisual Communication. Cultural diversity. Public policies. UNESCO.

Recebido em 10/11/2016 Aceito em 04/03/17 